\title{
The effectiveness of transforaminal epidural steroid injection in patients with radicular low back pain: Combination of pain provocation with effectiveness results
}

\author{
Emre Adıgüzel, ${ }^{1}$ Duygu Tecer, ${ }^{2}$ Ümüt Güzelküçük, ${ }^{1}$ Mehmet Ali Taşkaynatan, ${ }^{1}$ Arif Kenan Tan ${ }^{1}$ \\ ${ }^{1}$ Department of Physical Medicine and Rehabilitation, Gülhane Military Medical Academy, TAF Rehabilitation Center, Ankara, Turkey \\ ${ }^{2}$ Department of Physical Medicine and Rehabilitation, Division of Rheumatology, Medical Faculty of Gazi University, Ankara, Turkey \\ Received: February 2016 Accepted: June 2016
}

\begin{abstract}
Objectives: This study aims to investigate the efficacy of transforaminal epidural steroid injection (TFESI) on low back pain relief and functional impairments and whether pain provocation during injection has an effect on pain relief in mid-term.

Patients and methods: The study, which was conducted between September 2012 and September 2013, included 62 patients with low back pain (38 males, 24 females; median age 45 years; min 22 - max 88 years). All injections were applied under C-arm fluoroscopy guidance, using a mix of betamethasone and lidocaine. A $100 \mathrm{~mm}$ Visual Analog Scale (VAS) for pain, Oswestry Disability Index (ODI), and Short Form-36 were administered before the injection, and at post-injection second and $12^{\text {th }}$ weeks. Presence of any pain provocation was questioned during injection.

Results: The most frequent level of intervention was L5 level. Median initial VAS score was $80.0(50.0 ; 100.0)$ mm, which was measured as $45.0(0.0 ; 90.0) \mathrm{mm}$ and $30.0(0.0 ; 100.0) \mathrm{mm}$ at the post-injection second and $12^{\text {th }}$ weeks, respectively. Median initial ODI score was $25.0(9.0 ; 43.0)$ points, which was measured as $17.0(3.0 ; 38.0)$ and $12.5(1.0 ; 38.0)$ points at the post-injection second and $12^{\text {th }}$ weeks, respectively. All subgroup scores of SF-36 improved significantly during the follow-up period. We detected statistically significant improvements in the outcome measurements at the post-injection second and $12^{\text {th }}$ weeks $(\mathrm{p}<0.05)$. There were significant differences between patients with positive and moderate pain provocation in terms of VAS $(\mathrm{p}=0.004)$ and ODI $(\mathrm{p}=0.006)$ scores.

Conclusion: In this follow-up study, transforaminal epidural steroid injection was found to be effective in both the early period and in the mid-term. Pain provocation was not clinically predictive for better outcome according to the results.
\end{abstract}

Keywords: Effectiveness; epidural injection; low back pain; pain provocation.

Low back pain (LBP) is the most common cause of disabilities worldwide. ${ }^{[1]}$ According to different study methods and populations, the prevalence rate of LBP in the lifetime ranges from 40 to $70 \%{ }^{[2,3]}$ Patients with disc pathologies may have axial or radicular pain or both. Compared to solely axial pain, patients with radicular pain characteristics might have unfavorable treatment results, higher healthcare costs, and disabilities. ${ }^{[4]}$

Epidural steroid injections are one of the most commonly used interventions in radicular LBP. These injections can be performed using transforaminal, caudal or interlaminar routes. All of these routes have been found to be effective for pain relief due to disc herniations but the transforaminal epidural steroid injection (TFESI) is known to require the least injectate volume to reach the pathology site. In a systematic review by MacVicar et al., ${ }^{[5]}$ TFESI was reported to have moderate efficacy, with approximately $50 \%$ of pain relief in $70 \%$ of patients. In addition to pain relief, a surgery sparing effect has been attributed to TFESI. ${ }^{[6,7]}$

During epidural steroid injection, patients sometimes report pain provocation in their usual radicular pain sites and this is believed to provide more pain relief compared to patients without pain provocation. To date, there have been few studies observing pain provocation in TFESI and these studies have reported no significant difference. ${ }^{[8,9]}$

Corresponding author: Emre Adıgüzel, MD. Gülhane Askeri Tıp Akademisi Fiziksel Tıp ve Rehabilitasyon Anabilim Dalı, TSK Rehabilitasyon ve Bakım Merkezi, 06020 Etlik, Ankara, Turkey. e-mail: dremreadiguzel@gmail.com 
The frequency of disc pathologies over the lifetime and the effect of the pain on functional activities render a valid treatment procedure necessary. The above-mentioned interventions such as TFESI, caudal and interlaminar injections are some of the available treatment methods used for this aim. In the literature, different success rates have been reported for TFESI because of the confounding factors such as different sample sizes, patient preferences, medications used, and outcome measures. In one review, ${ }^{[5]}$ the success rate was reported as $70 \%$, while $50 \%$ of patients reported pain relief in another study. ${ }^{[10]}$

In this study, we aimed to investigate the efficacy of TFESI on LBP relief and functional impairments and whether pain provocation during injection has an effect on pain relief in the mid-term.

\section{PATIENTS AND METHODS}

A total of 62 patients ( 38 males, 24 females; median age 45 years; min 22 - max 88 years) were enrolled in this study, which was conducted at Turkish Armed Forces Rehabilitation Center Interventiona Pain Unit in a national rehabilitation setting between September 2012 and September 2013. The study design was approved by Gülhane Military Medical Academy Ethics Committee. All patients provided a signed written informed consent form before inclusion in the study. The study was conducted in accordance with the principles of the Declaration of Helsinki.

Inclusion criteria were (i) radicular LBP ongoing for at least three months, (ii) radicular pain concordant with magnetic resonance imaging (MRI) findings, and (iii) age $>18$ years. Patients with coagulation disorders, pregnancy or allergies to lidocaine, betamethasone or contrast medium were excluded.

We performed all injections in interventional pain unit of a national rehabilitation setting. We selected the injection level according to the affected nerve root, not according to the disc pathology itself. If an L4-5 intervertebral disc herniation involved the L5 nerve, we placed the needle between the L5 and S1 levels. If the $\mathrm{L} 4$ root was involved, we placed the needle between the L4 and L5 levels. We determined the affected nerve root according to electromyography (if any) and clinical findings.

We performed all injections using a subpedicular transforaminal technique. ${ }^{[1]}$ With the patient in the prone position and under sterile preparation, we advanced a $22 \mathrm{G}$ spinal needle into the neural foramen under fluoroscopy guidance. We used contrast medium of
1-2 $\mathrm{mL}$ (iohexol $300 \mathrm{mg} / \mathrm{mL}$ ) for both confirmation of the targeted nerve root and any intravasation of the medium. If there was no intravasation, we injected a mixture of $1 \mathrm{~mL}$ betamethasone $(6 \mathrm{mg})$ and $1 \mathrm{~mL}$ of lidocaine.

To assess whether pain was provoked during the injection, we questioned the patient about any pain provocation. If the patient reported pain provocation at the typical site of pain, we recorded positive pain provocation. If the patient felt pain provocation but it was not in the usual localization or it was a mild pain at the usual pain localization, we recorded moderate pain provocation. If there was no pain provocation, we recorded a negative response. After the injection, we monitored all patients for one hour in the clinic for any acute complications. On the day after the injection, we contacted the patient by telephone and asked about any complications.

We recorded demographic data and history of failed back surgery. We assessed pain with a Visual Analog Scale (VAS; $0=$ no pain, $10=$ the worst pain imaginable) before the injection and at the post-injection second and $12^{\text {th }}$ weeks. Visual Analog Scale is considered to be a valid measure of pain. ${ }^{[12]}$ The reliability of this instrument has been reported as $0.97-0.99 .^{[13]}$

We assessed patient satisfaction at the second and $12^{\text {th }}$ weeks with a 4-point Likert scale, asking the patient to rate as (i) no satisfaction, (ii) little satisfaction, (iii) moderate satisfaction, (iv) high satisfaction. We applied the Oswestry Disability Index (ODI) and Short Form-36 (SF-36) questionnaires before the injection, and at the second and $12^{\text {th }}$ weeks for functional patient assessment.

The ODI is a questionnaire that provides information about the patient's LBP and how this pain affects daily life. This questionnaire includes 10 sections about pain and related activities such as personal care, walking, lifting, standing, sleeping, sitting, social life, traveling and sexual life. Each section is scored from 0 to 5 points and a higher score indicates a higher level of disability. The maximum score is 50 points and the index value is obtained by multiplying the total points by two. This questionnaire has been demonstrated to be a valid measurement in patients with LBP. ${ }^{[14]}$ The reliability of this instrument has been reported as $0.83-0.99 .^{[15]}$

The SF-36 is a scale that includes eight scales and two summary scales. These are physical functioning, role limitations due to physical health, bodily pain, general health perceptions, vitality, social functioning, emotional problems, mental health and a physical and mental component summary. This instrument is also 
valid and reliable in spinal diseases. ${ }^{[16]}$ The reliability of this scale has been reported as $0.88-0.92 .^{[17]}$

All patients underwent physical examination before injection by the same practitioner. Two physicians independent of the intervention applied pain assessments, satisfaction ratings, ODI, and SF-36 questionnaires. We asked patients to report any complications after injection by telephone call or visit.

\section{Statistical analysis}

We used $G^{*}$ power program 3.1.9.2 (Heinrich Heine University, Dusseldorf, Germany) for statistical power analysis of variance (ANOVA). Fixed effects, special, main effects and interactions and type of power analysis were selected as post-hoc: compute achieved power-given $\alpha$, sample size, and effect size. With an $\alpha$ error level at 0.05 and a sample size of 62 patients, power of the current study was calculated as 0.98 .

Statistical analyses were performed with IBM SPSS for Windows version 22.0 software (IBM Corp., Armonk, NY, USA). Continuous variables with non-normal distribution were presented as median (minimum; maximum) and continuous variables with normal distribution were presented as mean \pm standard deviation. Qualitative variables were stated as number and percentage. The KolmogorovSmirnov test was used to determine the normality of data distribution. When the data was non-normally distributed, comparisons between two groups were conducted with Mann-Whitney U test. Two-factor ANOVA with repeated measures test was used for normally distributed data, and the Friedman test was used to compare follow-up VAS, ODI, and SF-36 results for non-normally distributed data. The Levene test was used to test the homogeneity of variances (all $\mathrm{p}>0.05$ ). When investigating the changes in measurements by pain provocation, the effect of time was adjusted using two-factor ANOVA test. Post-hoc analyses were performed using Tukey test. McNemar-Bowker test was used to analyze patient satisfaction. A Cohen's d calculation between second and $12^{\text {th }}$ week measurements was used to determine the effect sizes. To check reliability of ODI and SF-36 questionnaires for the sample of the current study, Cronbach alpha coefficient was used. A value of $\mathrm{p}<0.05$ was considered statistically significant.

\section{RESULTS}

Flow diagram of the study design is given in Figure 1. Nine patients (14.5\%) had failed back surgery, while four patients (6.5\%) had a history of TFESI.
According to the MRI findings, there was bulging in 21 patients $(33.9 \%)$, protrusion in 25 patients (40.3\%) and extrusion in 16 patients (25.8\%). Most of the injections $(54.8 \%, \mathrm{n}=34)$ were performed at L5 level. Five patients (8.1\%) underwent injection at S1 and three patients (4.8\%) at L4 levels. There were also injections applied at two levels. Injection was applied at L5 and S1 in 13 patients (21.0\%), at L4 and L5 in six (9.7\%), and at L4 and S1 in one patient (1.6\%).

Reliability of items in the ODI and SF-36 were high for this sample (Cronbach alpha $=0.89$ and 0.91, respectively). The results of patient assessments according to VAS pain, ODI, and SF-36 questionnaires are shown in Table 1. Transforaminal epidural steroid injection was significantly effective in all of these assessments at the second and the $12^{\text {th }}$ weeks (all $\mathrm{p}<0.001$, Friedman test). The effect sizes of VAS, ODI, and most of the SF-36 scores were large. There was no difference in follow-up outcome measurements in patients with failed back surgery when compared to other patients ( $>0.05$, Mann-Whitney $U$ test). Forty-six patients (74.2\%) reported moderate to high satisfaction at the post-injection $12^{\text {th }}$ week (Table 2). We detected a statistically significant difference between the second and $12^{\text {th }}$ week satisfaction rate assessments $(\mathrm{p}=0.03$, McNemar-Bowker test).

In the current study, the effect of pain provocation on VAS (Table 3, Figure 2), ODI, and SF-36 scores was also assessed. A two-factor ANOVA was conducted on the influence of two independent variables (pain provocation, time) on VAS scores. All effects were statistically significant. The main effect for pain provocation yielded an $\mathrm{F}$ ratio of $\mathrm{F}(2,17)=5.44$, $\mathrm{p}=0.005$, indicating significant difference between negative (53.0 \pm 24.4$)$, moderate $(60.0 \pm 23.6)$, and positive pain provocation groups $(47.6 \pm 29.1)$. The main effect for time yielded an $\mathrm{F}$ ratio of $\mathrm{F}(2,17)=57.8, \mathrm{p} \leq 0.001$, indicating a significance for time. The interaction effect was not significant, $F(4,17)=2.37, p=0.054$. Significance of the corrected model was $<0.001(\mathrm{~F}=22.9)$ and calculated partial eta squared value was 0.510 for this model. According to the measurements adjusted for the effect of time (week 0, 2 and 12), only positive and moderate provocation groups showed significant difference for VAS ( $p=0.004$, Tukey test) and ODI $(p=0.006$, Tukey test) scores. We found significant difference in social function, emotional role, mental health, and mental components summary subsets of SF-36 between positive and negative provocation groups (all $\mathrm{p}<0.05$, Tukey test). 


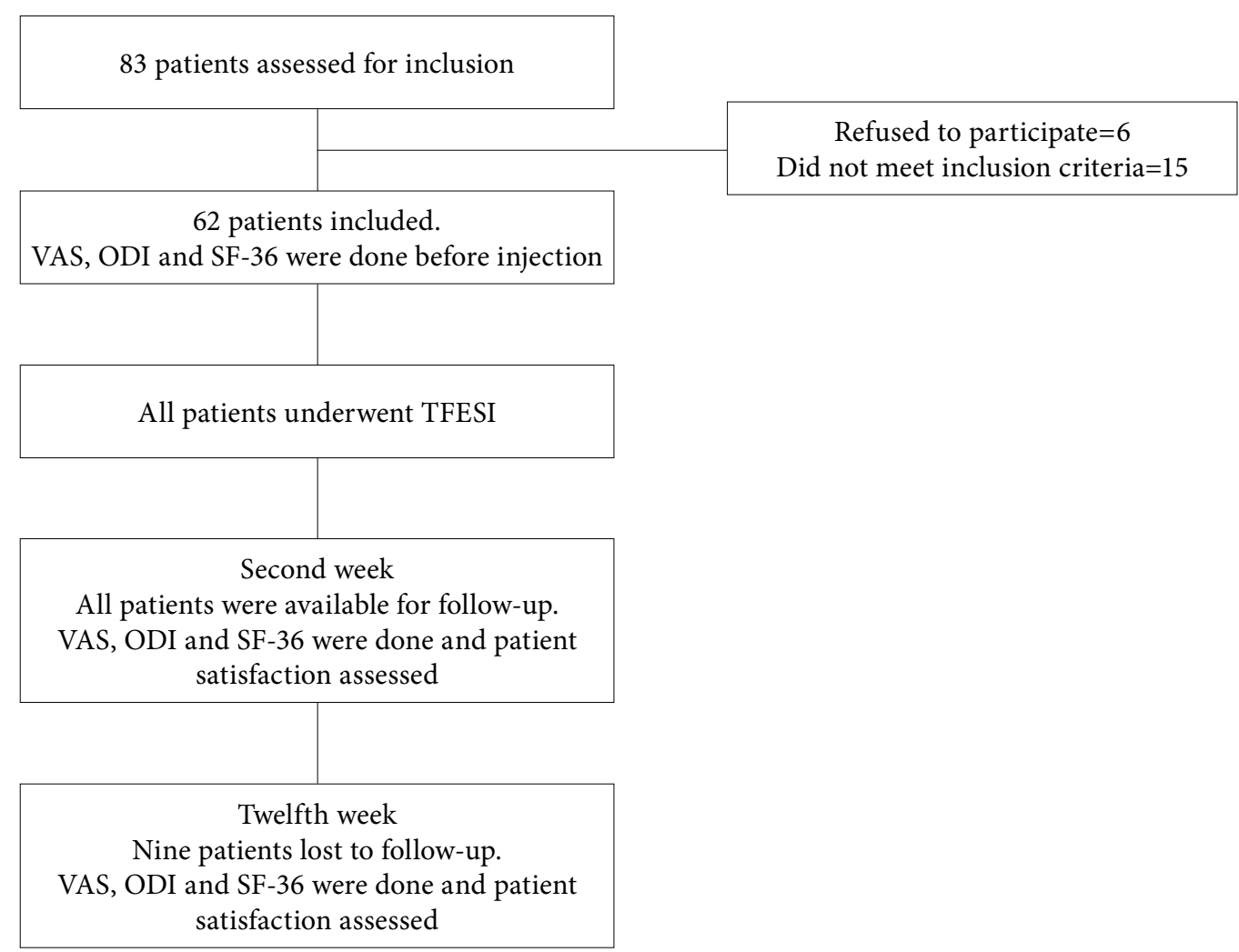

Figure 1. Flow diagram of study design. VAS: Visual Analog Scale; ODI: Oswestry disability index; SF-36: Short Form 36; TFESI: Transforaminal epidural steroid injection.

We recorded the complications after injections. Each of the following situations occurred in $1.6 \%$ of patients: vasovagal syncope, nausea, headache, injection site soreness, temporary urinary stress incontinence, hiccups, elevated blood sugar, and tinnitus. There was no major or permanent complication. No relationship was determined between adverse event occurrence and injection level ( $\mathrm{p}=0.3$, Chi-square test).

Table 1. Follow-up results of patients according to Visual Analog Scale pain, Oswestry disability index, and Short Form 36 questionnaires

\begin{tabular}{|c|c|c|c|c|c|c|c|c|}
\hline & \multicolumn{2}{|c|}{ Before injection } & \multicolumn{2}{|c|}{ Week 2} & \multicolumn{2}{|c|}{ Week 12} & \multirow[t]{2}{*}{$P^{*}$} & \multirow{2}{*}{$\begin{array}{c}\text { Effect size } \\
(\mathrm{d})^{\star *}\end{array}$} \\
\hline & Median & Min-Max & Median & Min-Max & Median & Min-Max & & \\
\hline Visual Analog Scale pain & 80.0 & $50.0-100.0$ & 45.0 & $0.0-90.0$ & 30.0 & $0.0-100.0$ & $<0.001$ & 2.02 \\
\hline Oswestry disability index & 25.0 & $9.0-43.0$ & 17.0 & $3.0-38.0$ & 12.5 & $1.0-38.0$ & $<0.001$ & 1.4 \\
\hline \multicolumn{9}{|l|}{ Short Form 36 scores } \\
\hline Physical functioning & 40.0 & $0.0-75.0$ & 60.0 & $0.0-95.0$ & 70.0 & $10.0-95.0$ & $<0.001$ & 1.35 \\
\hline Role, physical & 0.0 & $0.0-100.0$ & 25.0 & $0.0-100.0$ & 50.0 & $0.0-100.0$ & $<0.001$ & 1.29 \\
\hline Bodily pain & 22.0 & $0.0-62.0$ & 41.0 & $0.0-84.0$ & 62.0 & $0.0-100.0$ & $<0.001$ & 1.52 \\
\hline General health perceptions & 51.0 & $5.0-97.0$ & 57.0 & $15.0-100.0$ & 62.0 & $15.0-100.0$ & $<0.001$ & 0.41 \\
\hline Vitality & 50.0 & $5.0-95.0$ & 55.0 & $15.0-95.0$ & 55.0 & $10.0-90.0$ & $<0.001$ & 0.39 \\
\hline Social function & 43.75 & $0.0-100.0$ & 50.0 & $12.5-100.0$ & 0.0 & $25.0-100.0$ & $<0.001$ & 0.86 \\
\hline Role, emotional & 0.0 & $0.0-100.0$ & 33.3 & $0.0-100.0$ & 66.7 & $0.0-100.0$ & $<0.001$ & 0.84 \\
\hline Mental health & 68.0 & $24.0-100.0$ & 72.0 & $20.0-100.0$ & 72.0 & $20.0-100.0$ & $<0.001$ & 0.37 \\
\hline Physical components summary & 28.5 & $12.2-49.1$ & 36.2 & $22.5-54.2$ & 43.3 & 21.1-55.8 & $<0.001$ & 1.35 \\
\hline Mental components summary & 42.0 & $20.5-63.6$ & 45.5 & $22.6-63.4$ & 47.9 & $22.1-63.6$ & $<0.001$ & 0.42 \\
\hline
\end{tabular}

* Friedman test; ${ }^{*}$ Cohen's d; Min: Minimum; Max: Maximum. 
Table 2. Patient satisfaction rates

\begin{tabular}{|c|c|c|c|c|c|c|}
\hline & \multirow[t]{2}{*}{ Before injection } & \multicolumn{2}{|c|}{ Week 2} & \multicolumn{2}{|c|}{ Week 12} & \multirow[b]{2}{*}{$p$} \\
\hline & & $\mathrm{n}$ & $\%$ & $\mathrm{n}$ & $\%$ & \\
\hline Patient satisfaction & & & & & & $0.03^{\star}$ \\
\hline No satisfaction & NA & 5 & 8.1 & 9 & 14.5 & \\
\hline Little satisfaction & NA & 14 & 22.6 & 7 & 11.3 & \\
\hline Moderate satisfaction & NA & 35 & 56.5 & 28 & 45.2 & \\
\hline High satisfaction & NA & 8 & 12.9 & 18 & 29.0 & \\
\hline
\end{tabular}

\section{DISCUSSION}

In this study of 62 patients with radicular LBP who were treated with TFESI, we noted significant improvements in all outcome measures (numerical pain ratings, ODI, and SF-36 scores). At the end of the $12^{\text {th }}$ week, the efficacy of the procedure was still continuing.

In this study, the efficacy of TFESI in reducing pain due to disc pathologies and the VAS score changes was similar to previous studies. ${ }^{[10,18]}$ In addition to pain scores, we used the ODI and SF-36 scales as outcome measurements to define the functional effect of TFESI on daily activities. In a previous study, Ghahreman et al. ${ }^{[10]}$ also assessed pain relief and functional impairments with SF-36 and reported improvements in physical functioning, social functioning, bodily pain, and disability subgroups. In a retrospective study, Kaufmann et al. ${ }^{[19]}$ investigated the correlations between pain relief and functional status using Roland-Morris disability scores and demonstrated improvements in pain ratings and also disability scores. In the current study, both ODI scores and all subgroup scores of SF-36 improved significantly in the follow-up period. These improvements support the positive effects of TFESI in physical functions of daily life, as described in previous studies. ${ }^{[10,19]}$

The improvements in the clinical scales were also reflected in the patient satisfaction rates. A significant proportion of the patients was satisfied with the injection both in early period and midterm follow-up. Çetin et al. ${ }^{[20]}$ also assessed patient satisfaction and reported that $63.9 \%$ of patients had moderate and high satisfaction. At the $12^{\text {th }}$ week, the rate of moderate and high satisfaction of the patients $(74.6 \%)$ in our study was higher than that reported by Çetin et al. ${ }^{[20]}$

In the current study, we also attempted to define the effect of pain provocation on pain relief after injection. McCormick et al. ${ }^{[8]}$ and Plastaras et al. ${ }^{[9]}$ investigated this issue in larger groups of patients who underwent TFESI and reported that approximately $70 \%$ of the patients experienced concordant pain during the injection procedure, although concordant pain provocation did not predict the magnitude of pain relief in long-term follow-up. The results of the current study were in concordance with the data of previous TFESI studies ${ }^{[8,9]}$ regarding the patients with positive pain provocation and no pain provocation. The assessment method of pain provocation may have

Table 3. Visual Analog Scale pain rating changes according to pain provocation

\begin{tabular}{|c|c|c|c|c|}
\hline & VAS before injection & VAS Week 2 & VAS Week 12 & \\
\hline & Mean \pm SD & Mean \pm SD & Mean \pm SD & \\
\hline \multicolumn{5}{|l|}{ Pain provocation } \\
\hline Positive & $77.0 \pm 15.5$ & $41.8 \pm 18.3$ & $24.0 \pm 22.8$ & \\
\hline Moderate & $77.2 \pm 11.9$ & $52.7 \pm 17.3$ & $50.9 \pm 29.4$ & \\
\hline \multirow[t]{2}{*}{ Negative } & $75.4 \pm 15.3$ & $45.0 \pm 19.4$ & $38.8 \pm 20.8$ & \\
\hline & SS & MS & $\mathrm{F}$ & $p^{*}$ \\
\hline \multicolumn{5}{|l|}{ Source of variance } \\
\hline Time & 43183.561 & 21591.781 & 57.825 & $<0.001$ \\
\hline Pain provocation & 4069.675 & 2034.838 & 5.449 & 0.005 \\
\hline Interaction & 3550.506 & 21591.781 & 57.825 & 0.054 \\
\hline
\end{tabular}




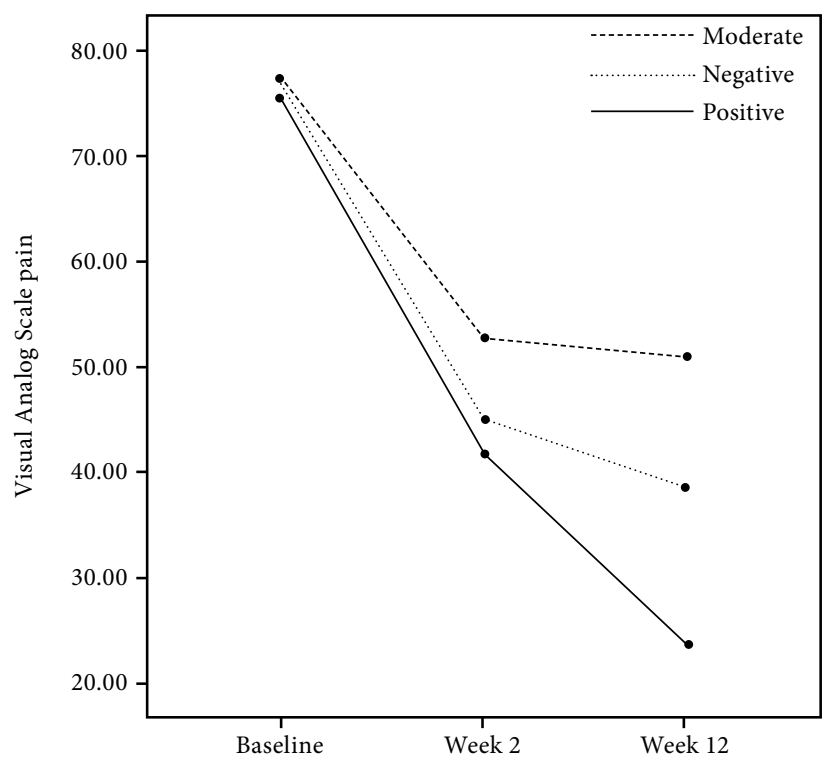

Figure 2. Visual Analog Scale pain ratings in the follow-up period according to pain provocation.

caused the difference between patients with positive and moderate pain provocation.

Previous studies have shown that TFESI may lead to major and minor adverse events. Major events include subdural and epidural hematoma, epidural abscess, discitis, and neurological deficits (paraplegia), while minor events include vasovagal syncope, increased pain, headache, injection site soreness, insomnia, flushing, and intravascular flow of the injectate. ${ }^{[5,21,22]}$ In the current study, adverse event rates were consistent with data described in the literature.

The current study has a number of limitations. There was no sham group to compare the efficacy of the injections. Although patients with more than three months of symptom duration were included, we did not assess the effect of symptom duration on follow-up results. In addition, there are some confounding factors such as psychiatric status, herniation level, location, size, and patients' body weight, which might have affected the results, but it was not possible to assess these factors because of the homogenous patient group. There are also strengths to this study. Patients with failed back surgery syndrome or those to whom TFESI was applied for a second time were not excluded. If it is assumed that these patients have intractable LBP, the results of the study are more reliable than those of other studies, which only included the patients with first application of TFESI or excluded patients with failed back surgery syndrome.
In conclusion, TFESI is effective in pain management of radicular LBP in the mid-term. The results of this study show that TFESI is effective not only in pain reduction but also in the improvement of activities of daily living. Future studies are necessary to decide whether pain provocation can provide clinically predictive data for better outcome.

\section{Declaration of conflicting interests}

The authors declared no conflicts of interest with respect to the authorship and/or publication of this article.

\section{Funding}

The authors received no financial support for the research and/or authorship of this article.

\section{REFERENCES}

1. Woolf AD, Pfleger B. Burden of major musculoskeletal conditions. Bull World Health Organ 2003;81:646-56.

2. Juniper M, Le TK, Mladsi D. The epidemiology, economic burden, and pharmacological treatment of chronic low back pain in France, Germany, Italy, Spain and the UK: a literaturebased review. Expert Opin Pharmacother 2009;10:2581-92.

3. Hoy D, Bain C, Williams G, March L, Brooks P, Blyth F, et al. A systematic review of the global prevalence of low back pain. Arthritis Rheum 2012;64:2028-37.

4. Konstantinou K, Dunn KM. Sciatica: review of epidemiological studies and prevalence estimates. Spine (Phila Pa 1976) 2008;33:2464-72.

5. MacVicar J, King W, Landers MH, Bogduk N. The effectiveness of lumbar transforaminal injection of steroids: a comprehensive review with systematic analysis of the published data. Pain Med 2013;14:14-28.

6. Riew KD, Yin Y, Gilula L, Bridwell KH, Lenke LG, Lauryssen $\mathrm{C}$, et al. The effect of nerve-root injections on the need for operative treatment of lumbar radicular pain. A prospective, randomized, controlled, double-blind study. J Bone Joint Surg [Am] 2000;82:1589-93.

7. Riew KD, Park JB, Cho YS, Gilula L, Patel A, Lenke LG, et al. Nerve root blocks in the treatment of lumbar radicular pain. A minimum five-year follow-up. J Bone Joint Surg [Am] 2006;88:1722-5.

8. McCormick Z, Margolis S, Temme K, Rivers E, Cameron SA, Smith MC, et al. Concordant pain provocation during transforaminal epidural steroid injection for lumbosacral radiculopathy: effect on pain outcome and predictive factors. Pain Physician 2015;18:19-26.

9. Plastaras CT HD, Sorosky BS, Houle TT. Pain reproduction during lumbosacral transforaminal epidural steroid injection does not affect outcome. J Back Musculoskelet Rehabil 2006;19:57-60.

10. Ghahreman A, Ferch R, Bogduk N. The efficacy of transforaminal injection of steroids for the treatment of lumbar radicular pain. Pain Med 2010;11:1149-68.

11. April C, Bogduk N, Dreyfuss P, Endres S, Govind J, Kraft M, et al, editors. Practice Guidelines for Spinal Diagnostic \& Treatment Procedures. 1st ed. San Francisco: International Spine Intervention Society; 2004. 
12. Gracely RH. Pain measurement. Acta Anaesthesiol Scand 1999;43:897-908.

13. Vela LI, Haladay DE, Denegar C. Clinical assessment of lowback-pain treatment outcomes in athletes. J Sport Rehabil 2011;20:74-88.

14. Fairbank JC, Pynsent PB. The Oswestry Disability Index. Spine (Phila Pa 1976) 2000;25:2940-52.

15. Roland M, Fairbank J. The Roland-Morris Disability Questionnaire and the Oswestry Disability Questionnaire. Spine (Phila Pa 1976) 2000;25:3115-24.

16. Beurskens AJ, de Vet HC, Köke AJ, van der Heijden GJ, Knipschild PG. Measuring the functional status of patients with low back pain. Assessment of the quality of four disease-specific questionnaires. Spine (Phila Pa 1976) 1995;20:1017-28.

17. Ware JE, Kosinski M, Keller JL, editors. SF-36 Physical and Mental Health Summary Scales: A User's Manual. Boston, MA: The Health Institute; 1994.

18. Vad VB, Bhat AL, Lutz GE, Cammisa F. Transforaminal epidural steroid injections in lumbosacral radiculopathy: a prospective randomized study. Spine (Phila $\mathrm{Pa}$ 1976) 2002;27:11-6.

19. Kaufmann TJ, Geske JR, Murthy NS, Thielen KR, Morris JM, Wald JT, et al. Clinical effectiveness of single lumbar transforaminal epidural steroid injections. Pain Med 2013;14:1126-33.

20. Çetin MF, Karaman H, Ölmez Kavak G, Tüfek A, Baysal Yildirim Z. Efficacy of transforaminal lumbar epidural steroid injections in patients with lumbar radiculopathy. [Article in Turkish] Agri 2012;24:77-84.

21. Kennedy DJ, Dreyfuss P, Aprill CN, Bogduk N. Paraplegia following image-guided transforaminal lumbar spine epidural steroid injection: two case reports. Pain Med 2009;10:1389-94.

22. Plastaras C, McCormick ZL, Garvan C, Macron D, Joshi A, Chimes G, et al. Adverse events associated with fluoroscopically guided lumbosacral transforaminal epidural steroid injections. Spine J 2015;15:2157-65. 